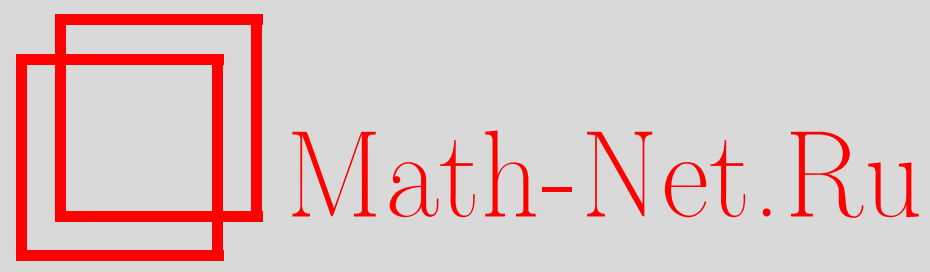

В. П. Серебряков, Условия квазирегулярности "чисто мнимого" сингулярного дифференциального оператора третьего порядка, Матем. заметки, 1997, том 61, выпуск 1, 149151

DOI: https://doi.org/10.4213/mzm1490

Использование Общероссийского математического портала Math-Net.Ru подразумевает, что вы прочитали и согласны с пользовательским соглашением http://www.mathnet.ru/rus/agreement

Параметры загрузки:

IP: 34.229 .108 .108

26 апреля 2023 г., $17: 36: 30$

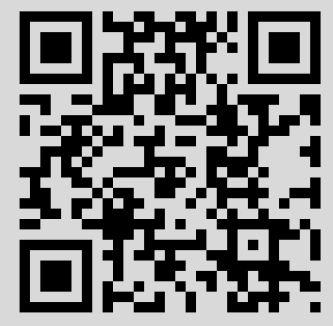




\section{УСЛОВИЯ КВАЗИРЕГУЛЯРНОСТИ “ЧИСТО МНИМОГО" СИНГУЛЯРНОГО ДИФФЕРЕНЦИАЛЬНОГО ОПЕРАТОРА ТРЕТЬЕГО ПОРЯДКА}

\section{В.П. Серебряков}

Рассмотрим формально самосопряженное сингулярное дифференциальное выражение третьего порядка с чисто мнимыми коэффициентами

$$
l[y]=-i y^{\prime \prime \prime}+\frac{i}{2}\left\{(p(x) y)^{\prime}+p(x) y^{\prime}\right\}, \quad 0 \leqslant x<\infty,
$$

где $p(x)$ - действительнозначная функция на полуоси $[0, \infty)$, абсолютно непрерывная на каждом сегменте $[0, b](0<b<\infty)$.

Обозначим через $\mathscr{L}$ минимальньй замкнутый симметрический оператор, порождаемый выражением $l[y]$ в пространстве $L_{2}[0, \infty)$.

Рассуждая так же, как и в $[1, \S 17]$, нетрудно показать, что

I) область определения $\mathscr{D}^{*}$ оператора $\mathscr{L}^{*}$, сопряженного с $\mathscr{L}$, состоит из функций $y \in L_{2}[0, \infty)$, абсолютно непрерьвных и имеющих абсолютно непрерывные производные первого и второго порядков на каждом сегменте $[0, b](0<b<\infty)$, для которых $l[y] \in L_{2}[0, \infty)$

II) если $y, z \in \mathscr{D}^{*}$, то существует конечньй предел

$$
\lim _{x \rightarrow \infty}[y, z]
$$

где

$$
[y, z]=-i\left(y^{\prime \prime} \bar{z}-y^{\prime} \bar{z}^{\prime}+y \bar{z}^{\prime \prime}\right)+i p y \bar{z}
$$

III) область определения $\mathscr{D}$ оператора $\mathscr{L}$ состоит из тех функций $y \in \mathscr{D}^{*}$, для которых $y(0)=y^{\prime}(0)=y^{\prime \prime}(0)=0$ и предел (1) равен нулю при каждом $z \in \mathscr{D}^{*}$.

Согласно [2], [3] индекс дефекта оператора $\mathscr{L}$ в зависимости от поведения его коэффициента $p(x)$ может принимать значения $\{1,2\},\{2,2\}$ или $\{3,3\}$.

Как известно, вопрос об индексе дефекта сингулярных дифференциальных операторов играет важную роль в спектральной теории дифференциальных операторов, в частности, в теории расширений симметрических операторов. Имеется немало работ, посвященных исследованию индекса дефекта операторов четного порядка (см., например, [1], [4]-[9]), а также библиографию, указанную в конце этих работ), для дифференциальных операторов нечетного порядка данньй вопрос изучен меньше.

Оператор $\mathscr{L}$ будем назьвать квазирегулярным, если его дефектные числа, как и в регулярном случае, равны 3 .

В настоящей заметке приведены условия на коэффициент $p$ для того, чтобы оператор $\mathscr{L}$ был квазирегулярным или, наоборот, неквазирегулярным. Для получения этих условий мы, в отличие, например, от [2], [3], не изучаем асимптотическое поведение решений уравнения $l[y]=\lambda y$ с комплексньм параметром $\lambda$, а используем свойства билинейной формы (2), ассоциированной с $\mathscr{L}$ (ср. с [4], [5], [7]-[9]).

Отметим, что если $p(x) \geqslant 0$ на $[0, \infty)$, то оператор $\mathscr{L}$ неквазирегулярньй, поскольку в этом случае решение $y(x)$ уравнения $l[y]=0(0 \leqslant x<\infty)$, удовлетворяющее начальным условиям $y(0)=y^{\prime}(0)=0, y^{\prime \prime}(0)=1$, не принадлежит $L_{2}[0, \infty)$. 
ТЕОремА. Пусть а-положительное действительное число, а $f$ и $g$-действительнозначные функиии, определенные на $[a, \infty)$ и обладающие свойствами

1) $f, f^{\prime}, f^{\prime \prime}$ и g абсолютно непрерывны на каждом сегменте $[a, b], a<b<\infty$;

2) $f$ не обращается в нуль на $[a, \infty)$;

3) функиии $g, g^{\prime} f+2 g f^{\prime}$ принадлежат $L_{2}[a, \infty)$.

Пусть $p=-f^{-2}\left(1+f^{\prime 2}-2 f f^{\prime \prime}\right)+g$ при $a \leqslant x<\infty$. Тогда

а) если $f \in L_{2}[a, \infty)$ и предел при $x \rightarrow \infty$ функции $g f^{2}$ равен нулю, то оператор $\mathscr{L}$ квазирегулярныи и;

b) если $f \notin L_{2}[a, \infty)$, то оператор $\mathscr{L}$ неквазирегулярный.

ДокАЗАТЕЛЬСтво. Для доказательства утверждения а) достаточно показать (см. $[1, \S 14])$, что коразмерность $\mathscr{D}$ в пространстве $\mathscr{D}^{*}$ не меньше 5.

Пусть

$$
\varphi(x)=f(x) \exp \left\{i \int_{a}^{x}(f(t))^{-1} d t\right\} .
$$

Пусть $\mathscr{D}_{a}^{*}-$ множество сужений на $[0, a]$ функций из $\mathscr{D}^{*}$, а $u_{n}-$ функции из $\mathscr{D}_{a}^{*}$, удовлетворяющие условиям (по поводу доказательства существования функций $u_{n}$ см. $[1, \S 17$, лемма 2 и ее доказательство])

$$
\begin{gathered}
u_{n}^{(k)}(0)=\delta_{n, k+1} \quad(n=1, \ldots, 5, \quad k=0,1,2) \\
u_{n}^{(k)}(a)=0, \quad u_{4}^{(k)}(a)=\varphi^{(k)}(a), \quad u_{5}^{(k)}(a)=\bar{\varphi}^{(k)}(a) \\
\left(n=1,2,3, \quad k=0,1,2, \quad \delta_{n, m}-\right.\text { символ Кронекера). }
\end{gathered}
$$

Положим

$$
y_{n}= \begin{cases}u_{n} & (0 \leqslant x<a, \quad n=1, \ldots, 5), \\ 0 & (a \leqslant x<\infty, \quad n=1,2,3), \\ \varphi & (a \leqslant x<\infty, n=4), \\ \bar{\varphi} & (a \leqslant x<\infty, \quad n=5) .\end{cases}
$$

Если $f \in L_{2}[a, \infty)$, то из построения функций $y_{n}$, условий 1$\left.), 3\right)$ доказываемой теоремы и утверждения I) следует, что все $y_{n}$ принадлежат $\mathscr{D}^{*}$.

Покажем, что $y_{n}(n=1, \ldots, 5)$ линейно независимы по модулю $\mathscr{D}$.

В самом деле, пусть

$$
\sum_{n=1}^{5} c_{n} y_{n} \in \mathscr{D},
$$

где $c_{n}$ - постоянные комплексные числа. Тогда согласно III) имеют место равенства

$$
\begin{gathered}
\sum_{n=1}^{5} c_{n} y_{n}^{(k)}(0)=0 \quad(k=0,1,2), \\
\lim _{x \rightarrow \infty}\left[\sum_{n=1}^{5} c_{n} y_{n}, y_{j}\right]=0 .
\end{gathered}
$$

Из (6), учитывая (3)-(5), находим $c_{1}=c_{2}=c_{3}=0$. 
Полагая в (7) последовательно $j=4,5$ и принимая во внимание (2)-(5), получаем, что если предел при $x \rightarrow \infty$ функции $g f^{2}$ равен нулю, то также $c_{4}=c_{5}=0$. Таким образом, $c_{n}=0(n=1, \ldots, 5)$ и утверждение а) теоремы доказано.

Утверждение b) теоремы можно получить, используя результаты работы [6].

СлЕДСТВИЕ. Пусть $0<a<\infty u$

1) функиии $p, p^{\prime}, p^{\prime \prime}$ абсолютно непрерывны на каждом сегменте $[a, b]$, $a<b<\infty$

2) $p<0$ на $[a, \infty)$;

3) функиии $p^{-2}\left(5 p^{\prime 2}-4 p p^{\prime \prime}\right),(-p)^{-7 / 2}\left(15{p^{\prime}}^{3}-18 p p^{\prime} p^{\prime \prime}+4 p^{2} p^{\prime \prime \prime}\right)$ принадлежсат $L_{2}[a, \infty)$.

Тогда

a) $\operatorname{ecлu}(-p)^{-1 / 2} \in L_{2}[a, \infty) u$

$$
\lim _{x \rightarrow \infty} p^{-3}\left(5 p^{2}-4 p p^{\prime \prime}\right)=0
$$

то оператор $\mathscr{L}$ квазирегулярный;

в) если $(-p)^{-1 / 2} \notin L_{2}[a, \infty)$, то оператор $\mathscr{L}$ неквазирегулярный.

ДокАЗАТЕльство. Для доказательства достаточно в установленной теореме взять

$$
f=(-p)^{-1 / 2}, \quad g=p^{-1} p^{\prime \prime}-\frac{5}{4} p^{-2} p^{2}
$$

Московский государственный университет

Поступило

им. М.В. Ломоносова

02.08 .94

Исправленный вариант

21.09 .95

\section{СПИСОК ЦИТИРОВАННОЙ ЛИТЕРАТУРЫ}

1. Наймарк М.А. Линейные дифференциальные операторы. М.: Наука, 1969. 2. Коган В.И., Рофе-Бекетов Ф.С. // Математическая физика и функциональный анализ. II. Харьков, 1971. С. 45-60. 3. Unsworth K. // Quart. J. Math. Ser. 2. 1973. V. 24. № 94. P. 177-188. 4. Everitt W. N. // Quart. J. Math. Ser. 2. 1972. V. 23. № 89. P. 193-196. 5. Easthman M.S.P. // J. London Math. Soc. Ser. 2. 1974. V. 8. P. 427-437. 6. Мирзоев К. А. // УМН. 1985. Т. 40. №1(241). С. 207-208. 7. Мирзоев К. А. // Матем. заметки. 1985. Т. 37. №5. С. 712-716. 8. Серебряков В.П. // Матем. заметки. 1988. Т. 44. № 6. С. 833-842. 9. Серебряков В. П. // Изв. вузов. Матем. 1995. № 3(394). С. 45-50. 